The explicitly correlated same number of optimized parameters (SNOOP-F12) scheme for calculating intermolecular interaction energies

Troels Hels Rasmussen, Yang Min Wang, Thomas Kjærgaard, and Kasper Kristensen

Citation: The Journal of Chemical Physics 144, 204102 (2016); doi: 10.1063/1.4950846

View online: http://dx.doi.org/10.1063/1.4950846

View Table of Contents: http://aip.scitation.org/toc/jcp/144/20

Published by the American Institute of Physics

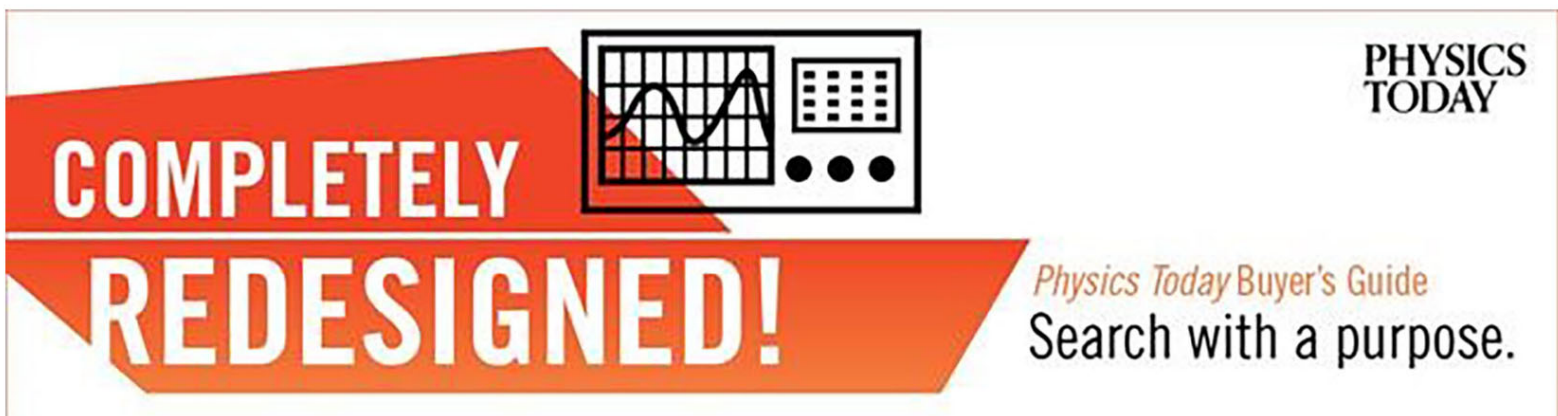




\title{
The explicitly correlated same number of optimized parameters (SNOOP-F12) scheme for calculating intermolecular interaction energies
}

\author{
Troels Hels Rasmussen, ${ }^{\text {a) }}$ Yang Min Wang, Thomas Kjærgaard, and Kasper Kristensen ${ }^{\text {b) }}$ \\ Department of Chemistry, qLEAP Center for Theoretical Chemistry, Aarhus University, Langelandsgade 140, \\ DK-8000 Aarhus C, Denmark
}

(Received 2 March 2016; accepted 6 May 2016; published online 23 May 2016)

\begin{abstract}
We augment the recently introduced same number of optimized parameters (SNOOP) scheme [K. Kristensen et al., J. Chem. Phys. 142, 114116 (2015)] for calculating interaction energies of molecular dimers with an F12 correction and generalize the method to enable the determination of interaction energies of general molecular clusters. The SNOOP, uncorrected (UC), and counterpoise (CP) schemes with/without an F12 correction are compared for the S22 test set of Jurečka et al. [Phys. Chem. Chem. Phys. 8, 1985 (2006)] — which consists of 22 molecular dimers of biological importance - and for water and methane molecular clusters. The calculations have been performed using the Resolution of the Identity second-order Møller-Plesset perturbation theory method. We conclude from the results that the SNOOP scheme generally yields interaction energies closer to the complete basis set limit value than the UC and CP approaches, regardless of whether the F12 correction is applied or not. Specifically, using the SNOOP scheme with an F12 correction yields the computationally most efficient way of achieving accurate results at low basis set levels. These conclusions hold both for molecular dimers and more general molecular clusters. Published by AIP Publishing. [http://dx.doi.org/10.1063/1.4950846]
\end{abstract}

\section{INTRODUCTION}

One of the main goals of computational chemistry is the accurate determination of intermolecular interaction energies of molecular dimers as well as more complex molecular clusters with applications in diverse fields, such as biochemistry and materials sciences. In standard quantum chemical calculations using finite atom-centered one-electron basis sets, any calculated total molecular energy will be subject to a basis set incompleteness error (BSIE) compared to the complete basis set (CBS) limit value of the energy. Consequently, an interaction energy calculated as the difference between the energy of an interacting $A B$ system and two noninteracting $A$ and $B$ monomers will also suffer from BSIE. An additional error source is introduced, since, as the two monomers approach each other, monomer $A$ in the dimer can lower its energy by using the basis functions of monomer $B$ (and vice versa). This effect, which is denoted the basis set superposition error (BSSE), ${ }^{1}$ is an artifact of using an incomplete one-electron basis, and it leads to an artificial attraction between the two monomers in addition to the physical interactions that one is trying to describe. BSIE and BSSE both disappear in the CBS limit.

The most common approach for correcting for BSSE is the counterpoise (CP) approach by Boys and Bernardi, ${ }^{2}$ where the calculation of monomer $A$ is carried out using the basis functions on monomer $A$ as well as the ghost basis functions of monomer $B$ positioned at the dimer geometry (and

\footnotetext{
a)Electronic mail: troels.hels.rasmussen@ post.au.dk

b)Electronic mail: kasperk@chem.au.dk
}

vice versa). However, the $\mathrm{CP}$ correction does not necessarily bring the calculated interaction energy closer to the CBS limit, ${ }^{3,4}$ because it corrects only for the BSSE and not the BSIE, which is often of opposite sign, and the theoretical and practical validity of the $\mathrm{CP}$ approach have been debated extensively in the literature. ${ }^{3-16}$ Several alternatives to the standard $\mathrm{CP}$ approach have been suggested, including the virtual CP (VCP) approach, ${ }^{17,18}$ scaled CP corrections, ${ }^{19-21}$ the semi-empirical geometrical $\mathrm{CP}$ approach, ${ }^{22}$ as well as schemes where the BSSE is eliminated a priori, such as symmetry-adapted perturbation theory (SAPT), ${ }^{23,24}$ the chemical Hamiltonian approach, ${ }^{25,26}$ the strictly monomermolecular orbital approach, ${ }^{27}$ and the constrained dimer function approach. ${ }^{28}$ Nonetheless, most practical applications continue to use the $\mathrm{CP}$ procedure.

Interaction energies calculated without employing ghost functions in the monomer calculations will be referred to as uncorrected (UC) interaction energies. For typical molecular dimers dominated by hydrogen bonds, dipoledipole interactions, and dispersion forces, UC interaction energies calculated using a given finite basis set are usually observed to be too attractive, whereas $\mathrm{CP}$-corrected interaction energies are not attractive enough (compared to the CBS limit). It turns out that the number of wave function parameters is smaller/larger for the noninteracting $A+B$ system than for the interacting $A B$ system when the $\mathrm{UC} / \mathrm{CP}$ approaches are used. In acknowledgment of this observation, we have recently proposed the Same Number Of Optimized Parameters (SNOOP) approach, ${ }^{29}$ where it is imposed that the number of non-redundant Hartree-Fock (HF) optimization parameters is the same for the noninteracting $A+B$ system as for the interacting $A B$ system to ensure a balanced description of the 
interaction. This, in turn, implies that the SNOOP interaction energy by construction is sandwiched between the UC and CP interaction energies.

As described above, BSIE is present in any conventional quantum chemical calculation. In fact, the BSIE of the total dimer energy may easily be an order of magnitude larger than the interaction energy itself when typical basis sets of double- or triple- $\zeta$ quality are employed. ${ }^{29}$ Consequently, the determination of the interaction energy as the difference between the dimer energy and the monomer energies relies on a remarkable error cancellation. The main reason for the sizable BSIE is the inability of the wave function to represent the Coulomb cusp, ${ }^{30}$ because conventional wave functions expressed in terms of Slater determinants do not satisfy Kato's cusp condition. ${ }^{31}$ However, this condition may be satisfied by devising methods which explicitly incorporate the inter-electronic distance operator into the wave-function ansatz. ${ }^{32-35}$ Such methods are referred to as explicitly correlated or F12 methods; for recent reviews, see Refs. 36-38. The augmentation of conventional correlated wave function models with an F12 correction ultimately allows for an efficient description of the Coulomb cusp and thus a significant lowering of the BSIE, which renders the F12 technology a very useful tool for the accurate determination of intermolecular interaction energies. ${ }^{39-46}$

In this work we augment the SNOOP method with an F12 correction and compare the performances of the UC, CP, and SNOOP approaches with and without the F12 correction. Conceptually, the F12 correction approximately describes the components of the wave function outside the computational basis, and it is therefore considerably different for the UC, CP, and SNOOP monomer calculations, for which the molecular orbital (MO) spaces of the conventional correlated calculation are different in size. We calculate interaction energies for the dimers of the $\mathrm{S} 22$ test set, ${ }^{47}$ and we also extend the SNOOP philosophy to the determination of intermolecular interaction energies for molecular clusters containing more than two interacting molecules. The calculations were carried out using the Resolution of the Identity second-order MøllerPlesset perturbation theory (RIMP2) method, and density fitting techniques ${ }^{48-53}$ were used to reduce the computational costs of evaluating integrals for both the MP2 ${ }^{54}$ and $F 12^{55}$ contributions.

The SNOOP scheme may be viewed as a variant of the VCP approach where the HF orbitals for monomer $A$ are fully optimized in the orbital space comprising the basis functions of monomer A as well as the virtual orbitals of monomer $B$. This is equivalent to the VCP variant used by Gutowski and Chałasińki to study the helium dimer. ${ }^{12}$ Using the so-called $\mathrm{CR} n \mathrm{DS} m$ basis sets specially designed for their investigation of the helium dimer, they demonstrated that the standard CP approach was superior to the VCP scheme. In this work, however, we consider the aug-cc-pVXZ $(X=D, T, Q, \ldots)$ basis sets $^{56}$ and show that for a wide variety of molecular dimers and clusters containing hydrogen, carbon, nitrogen, and oxygen atoms, the SNOOP interaction energies are closer to the CBS limit values than interaction energies calculated using the $\mathrm{CP}$ scheme, regardless of whether the F12 correction is applied or not.
In Section II we summarize the SNOOP scheme and discuss the implications of extending the UC, CP, and SNOOP schemes with an F12 correction. Section III contains computational details, and the results for interaction energies of dimers and molecular clusters are given in Section IV. Section V contains some concluding remarks.

\section{THEORY}

In this section we describe the theoretical framework used for calculating the presented intermolecular interaction energies. In Section II A we summarize the UC, CP, and SNOOP approaches, noting that a more detailed description of the SNOOP scheme is given in Ref. 29. In Section II B we describe how the UC, CP, and SNOOP interaction energies may be augmented with an F12 correction.

\section{A. UC, CP, and SNOOP approaches}

When two monomers $A$ and $B$ interact to form a dimer $A B$, the interaction energy, $\Delta E_{A B}$, is defined as the difference between the total energy of $A B, E_{A B}$, and the total energy of the noninteracting monomers, $E_{A}+E_{B}$. In this work, we consider only the electronic contribution to the interaction energy, i.e., we do not consider changes in the zero point vibrational energy or the relaxation effects associated with the difference between the geometry of the isolated monomer and the geometry of the monomer in the interacting system. In the following the noninteracting monomers $A$ and $B$ will be collectively referred to as $A+B$.

Conventional quantum chemical calculations employ atom-centered basis functions (atomic orbitals, AOs), and each AO may thus be assigned to either monomer $A$ or $B$. Imagine now that two separate $\mathrm{HF}$ calculations are carried out for monomers $A$ and $B$, i.e., the HF calculation for monomer $A$ uses only AOs assigned to $A$, and similarly for monomer B. The set of occupied/virtual MOs resulting from these HF calculations will be denoted $O_{A} / \mathcal{V}_{A}$ and $O_{B} / \mathcal{V}_{B}$ for monomers $A$ and $B$, respectively, while the number of orbitals in those sets are denoted $o_{A} / v_{A}$ and $o_{B} / v_{B}$. The $O_{A} \cup \mathcal{V}_{A}$ space thus spans the same space as the AOs assigned to monomer $A$. The calculation for the interacting dimer $A B$ is performed using the total set of AOs, which is spanned by $O_{A} \cup \mathcal{V}_{A} \cup O_{B} \cup \mathcal{V}_{B}$. In the UC approach for calculating interaction energies, the monomer energy $E_{A}$ is calculated using the $O_{A} \cup \mathcal{V}_{A}$ orbital space. Using curly braces to highlight the orbital basis used in each calculation, the UC interaction energy, $\Delta E_{A B}^{\mathrm{UC}}$, may thus be written as

$\Delta E_{A B}^{\mathrm{UC}}=E_{A B}^{\left\{O_{A} \cup \mathcal{V}_{A} \cup O_{B} \cup \mathcal{V}_{B}\right\}}-E_{A}^{\left\{O_{A} \cup \mathcal{V}_{A}\right\}}-E_{B}^{\left\{O_{B} \cup \mathcal{V}_{B}\right\}}$.

It is well-known that the interaction energy defined by Eq. (1) suffers from BSSE, i.e., the monomer $A$ in the $A B$ complex can lower its energy by using the basis functions of monomer $B$ (and vice versa). This leads to an artificial attraction of the two monomers in addition to the physical attraction (or repulsion) that one is trying to describe. The most commonly used approach for approximately correcting for the BSSE is the CP approach, ${ }^{2}$ where each monomer calculation uses the 
full basis, i.e., using our notation, the $\mathrm{CP}$-corrected interaction energy may be written as

$$
\begin{aligned}
\Delta E_{A B}^{\mathrm{CP}}= & E_{A B}^{\left\{O_{A} \cup \mathcal{V}_{A} \cup O_{B} \cup \mathcal{V}_{B}\right\}}-E_{A}^{\left\{O_{A} \cup \mathcal{V}_{A} \cup O_{B} \cup \mathcal{V}_{B}\right\}} \\
& -E_{B}^{\left\{O_{A} \cup \mathcal{V}_{A} \cup O_{B} \cup \mathcal{V}_{B}\right\}} .
\end{aligned}
$$

Assuming that the $A \cdots B$ interaction is attractive, the $\Delta E_{A B}^{\mathrm{CP}}$ interaction energy is less negative than $\Delta E_{A B}^{\mathrm{UC}}$, because the $\mathrm{CP}$ monomer energies are lowered compared to the UC ones due to the larger degree of freedom in the wave function parameters.

In practical calculations using a finite basis, it is often observed that $\Delta E_{A B}^{\mathrm{UC}}$ is too negative, while $\Delta E_{A B}^{\mathrm{CP}}$ is not negative enough compared to the CBS limit value for the interaction energy. It has been shown that the CP-corrected interaction energy for a given basis set is consistent with the result obtained from a SAPT calculation ${ }^{11,12}$ and that the dispersion component of the interaction energy calculated using a finite basis set is generally not negative enough. Thus, when the $\mathrm{CP}$ correction is applied to correct for BSSE, the resulting interaction energy tends to be too repulsive compared to the CBS limit value because the BSSE has a different sign than the BSIE, which is not improved by the CP correction. Consequently, even though the $\mathrm{CP}$ approach is consistent with a SAPT analysis, there is no guarantee that the CP-corrected interaction energy calculated using a finite basis set is closer to the CBS limit value than the corresponding UC interaction energy. In fact, in practical calculations the $\mathrm{CP}$ correction is sometimes observed to worsen the UC result. ${ }^{3,4}$

An alternative to the $\mathrm{UC}$ and $\mathrm{CP}$ approaches is to impose a balance between the dimer and monomer calculations in terms of the number of wave function parameters employed in the calculations. The number of non-redundant wave function parameters used to describe the interacting $A B$ dimer is larger (smaller) than the corresponding number of parameters for the noninteracting $A+B$ system described at the UC (CP) level. As an attempt to impose a balanced description, we have suggested the SNOOP scheme, ${ }^{29}$ where the number of wave function parameters for the noninteracting $A+B$ system is the same as for the $A B$ dimer. Thus, if the number of parameters is denoted $P$, the following set of inequalities hold:

$$
P_{A+B}^{\mathrm{UC}}<P_{A+B}^{\mathrm{SNOOP}}=P_{A B}<P_{A+B}^{\mathrm{CP}} .
$$

The equality in Eq. (3) is satisfied if the HF optimization for monomer $A$ is carried out using a basis containing both the orbital space for $A$ (spanned by $O_{A} \cup \mathcal{V}_{A}$ ) and the virtual orbitals for $B$ obtained in the UC calculations $\left(\mathcal{V}_{B}\right)$, and vice versa for monomer $B .^{29}$ The SNOOP interaction energy may thus be written as

$$
\begin{aligned}
\Delta E_{A B}^{\mathrm{SNOOP}}= & E_{A B}^{\left\{O_{A} \cup \mathcal{V}_{A} \cup O_{B} \cup \mathcal{V}_{B}\right\}}-E_{A}^{\left\{O_{A} \cup \mathcal{V}_{A} \cup \mathcal{V}_{B}\right\}} \\
& -E_{B}^{\left\{O_{B} \cup \mathcal{V}_{B} \cup \mathcal{V}_{A}\right\}} .
\end{aligned}
$$

From Eq. (3) it follows that each of the SNOOP monomer energies-and thus the SNOOP interaction energy-is sandwiched between the interaction energies obtained using the UC and $\mathrm{CP}$ approaches, i.e.,

$$
\begin{gathered}
E_{X}^{\mathrm{UC}} \geq E_{X}^{\mathrm{SNOOP}} \geq E_{X}^{\mathrm{CP}} \quad(X=A, B), \\
\Delta E_{A B}^{\mathrm{UC}} \leq \Delta E_{A B}^{\mathrm{SNOOP}} \leq \Delta E_{A B}^{\mathrm{CP}} .
\end{gathered}
$$

The above discussion is restricted to molecular dimers, but the generalization to molecular clusters containing more than two interacting monomers is straightforward as shown in the Appendix.

\section{B. The F12 correction}

In the limit where the basis set on the individual monomers approach completeness, the UC, CP, and SNOOP interaction energies all approach the same result-the CBS limit value. The CBS result may be approached systematically, e.g., by increasing the cardinal number of the aug-cc-pVXZ $(\mathrm{X}=\mathrm{D}, \mathrm{T}, \mathrm{Q}, \ldots)$ basis sets. ${ }^{56}$ However, the slow convergence of the correlation energy with increasing basis sets ${ }^{57,58}$ poses a problem in practical calculations. The primary cause of this slow convergence is that conventional wave functions do not satisfy Kato's cusp condition. This condition can be fulfilled by including the inter-electronic distance operator into the wave-function ansatz, and such methods are referred to as F12 methods. Since the technical aspects of F12 theory have been reviewed in detail elsewhere, ${ }^{36-38}$ we consider here only some basic aspects of F12 theory that will highlight the formal differences between the UC, CP, and SNOOP schemes. In this work we use the RIMP2 model, and the F12 correction is calculated using the so-called approximation $3 C^{58,59}$ and the fixed amplitude ansatz, ${ }^{60}$ but we note that the conceptual considerations are applicable to a general coupled-cluster wave function model and a general F12 scheme.

We may write the RIMP2-F12 energy as a sum of the conventional RIMP2 energy calculated using a given regular MO basis and an F12 correction

$$
E_{\mathrm{RIMP} 2-\mathrm{F} 12}=E_{\mathrm{RIMP} 2}+E_{\mathrm{F} 12} \text {. }
$$

The two-electron F12 basis functions (geminals) are central to F12 theory, and a discussion of the geminals will elucidate the conceptual differences between the three approaches within an F12 context. In the following we let $i, j$ $(a, b)$ denote occupied (virtual) MO indices. A geminal $\left|w_{i j}\right\rangle$ may be written as

$$
\left|w_{i j}\right\rangle=\hat{Q}_{12} f_{12}\left|\phi_{i}\left(\mathbf{r}_{1}\right) \phi_{j}\left(\mathbf{r}_{2}\right)\right\rangle .
$$

Here, $\phi_{i}\left(\mathbf{r}_{1}\right)$ and $\phi_{j}\left(\mathbf{r}_{2}\right)$ are occupied MOs evaluated for electron coordinates 1 and 2 , while $\hat{Q}_{12}$ is a projection operator that ensures strong orthogonality of the geminals to any product of MOs within the computational basis ${ }^{37}$

$$
\hat{Q}_{12}=\left(1-\hat{O}_{1}\right)\left(1-\hat{O}_{2}\right)-\hat{V}_{1} \hat{V}_{2},
$$

where $\hat{O}_{m}$ and $\hat{V}_{m}$ projects onto the occupied and virtual MO spaces for electronic coordinate $m$, respectively,

$$
\begin{aligned}
& \hat{O}_{m}=\sum_{i}\left|\phi_{i}\left(r_{m}\right)\right\rangle\left\langle\phi_{i}\left(r_{m}\right)\right|, \\
& \hat{V}_{m}=\sum_{a}\left|\phi_{a}\left(r_{m}\right)\right\rangle\left\langle\phi_{a}\left(r_{m}\right)\right| .
\end{aligned}
$$

The inter-electronic distance function $f_{12}$ can be written as ${ }^{61-63}$

$$
f_{12}=-\frac{1}{\gamma} e^{-\gamma r_{12}},
$$


where $r_{12}$ is the inter-electronic distance between electrons 1 and 2 , and $\gamma$ is a basis set dependent parameter, which we have chosen in accordance with the recommendations of Ref. 64.

When the $f_{12}$ operator works on $\left|\phi_{i}\left(\mathbf{r}_{1}\right) \phi_{j}\left(\mathbf{r}_{2}\right)\right\rangle$, a new two-electron function is generated, which may be viewed as a combination of two-electron states in the complete basis, while the $\hat{Q}_{12}$ operator removes components from the regular MO basis. The geminals $\left|w_{i j}\right\rangle$ in Eq. (8) thus represent a two-electron basis outside the regular MO basis. Even with a relatively small regular basis, the introduction of geminals enables an efficient description of wave function components outside the regular MO basis, which primarily represent the Coulomb cusps in the wave function.

Let us compare the occupied and virtual projection operators in Eq. (10) for the UC, CP, and SNOOP schemes in detail. The dimer energy $E_{A B}$ is the same for the three schemes, and we will focus on monomer $A$ in the following analysis (the corresponding analysis for monomer $B$ is obtained by reversing $A$ and $B$ below). We use the following notation for the occupied and virtual orbital spaces used in the monomer $A$ calculations for the three schemes:

- $O_{A} / \mathcal{V}_{A}$ : The set of occupied/virtual orbitals $\left\{\phi_{i}^{\mathrm{UC}}\right\} /$ $\left\{\phi_{a}^{\mathrm{UC}}\right\}$ [dimension $o_{A} / v_{A}$ ] resulting from a $\mathrm{HF}$ optimization of monomer $A$ using basis functions on monomer $A$.

- $O_{A}^{\mathrm{CP}} / \mathcal{V}_{A}^{\mathrm{CP}}$ : The set of occupied/virtual orbitals $\left\{\phi_{i}^{\mathrm{CP}}\right\} /\left\{\phi_{a}^{\mathrm{CP}}\right\}\left[\right.$ dimension $\left.o_{A} /\left(v_{A}+v_{B}+o_{B}\right)\right]$ resulting from a HF optimization of monomer $A$ in the $O_{A} \cup \mathcal{V}_{A} \cup \mathcal{O}_{B} \cup \mathcal{V}_{B}$ space.

- $O_{A}^{\mathrm{SNOOP}} / \mathcal{V}_{A}^{\mathrm{SNOOP}}:$ The set of occupied/virtual orbitals $\left\{\phi_{i}^{\mathrm{SNOOP}}\right\} /\left\{\phi_{a}^{\mathrm{SNOOP}}\right\}\left[\right.$ dimension $\left.o_{A} /\left(v_{A}+v_{B}\right)\right]$ resulting from a $\mathrm{HF}$ optimization of monomer $A$ in the $O_{A} \cup \mathcal{V}_{A} \cup \mathcal{V}_{B}$ space.

We note that not only are the dimensions of the virtual spaces different for the three schemes, the occupied orbitals are also different because they have been optimized in different spaces.
The occupied and virtual projection operators for monomer $A$ for the three schemes may thus be written as

$$
\begin{aligned}
\hat{O}_{m, A}^{\mathrm{M}} & =\sum_{i \in O_{A}^{\mathrm{M}}}\left|\phi_{i}^{\mathrm{M}}\left(r_{m}\right)\right\rangle\left\langle\phi_{i}^{\mathrm{M}}\left(r_{m}\right)\right|, \\
\hat{V}_{m, A}^{\mathrm{M}} & =\sum_{a \in \mathcal{V}_{A}^{\mathrm{M}}}\left|\phi_{a}^{\mathrm{M}}\left(r_{m}\right)\right\rangle\left\langle\phi_{a}^{\mathrm{M}}\left(r_{m}\right)\right|,
\end{aligned}
$$

and, using Eq. (8), the resulting geminals become

$$
\begin{aligned}
\left|w_{i j, A}^{\mathrm{M}}\right\rangle= & \left(\left(1-\hat{O}_{1, A}^{\mathrm{M}}\right)\left(1-\hat{O}_{2, A}^{\mathrm{M}}\right)-\hat{V}_{1, A}^{\mathrm{M}} \hat{V}_{2, A}^{\mathrm{M}}\right) \\
& \times f_{12}\left|\phi_{i}^{\mathrm{M}}\left(\mathbf{r}_{1}\right) \phi_{j}^{\mathrm{M}}\left(\mathbf{r}_{2}\right)\right\rangle,
\end{aligned}
$$

with $\mathrm{M}=\mathrm{UC}, \mathrm{CP}$, or SNOOP and $O_{A}^{\mathrm{UC}} \equiv O_{A}, \mathcal{V}_{A}^{\mathrm{UC}} \equiv \mathcal{V}_{A}$.

Conceptually, Eqs. (12)-(14) may be interpreted in the following manner. As discussed above, $f_{12}\left|\phi_{i}^{\mathrm{M}}\left(\mathbf{r}_{1}\right) \phi_{j}^{\mathrm{M}}\left(\mathbf{r}_{2}\right)\right\rangle$ (M = UC, CP, SNOOP) contains components of two-electron functions both within and outside the regular MO basis. The projection operator in Eq. (14) projects out MOs that have already been taken into account by the conventional RIMP2 calculation for monomer $A$, and the complementary virtual space outside the regular MO basis, $\tilde{V}_{A}$, which is approximately represented by the geminals in Eq. (14), is thus different for the three schemes. For the CP approach, the complementary virtual space for monomer $A, \tilde{V}_{A}^{\mathrm{CP}}$, only contains orbitals that are outside the dimer MO basis $\left(O_{A} \cup\right.$ $\mathcal{V}_{A} \cup O_{B} \cup \mathcal{V}_{B}$ ), while $\tilde{\mathcal{V}}_{A}^{\text {SNOOP }}$ may also contain components of $O_{B}$, and $\tilde{V}_{A}^{\mathrm{UC}}$ may contain components of $O_{B}$ and $\mathcal{V}_{B}$. In other words, the orbitals of the complementary $\tilde{V}_{A}^{\text {CP }}, \tilde{V}_{A}^{\text {SNOOP }}$, and $\tilde{V}_{A}^{\mathrm{UC}}$ spaces represented by the geminals in Eq. (14) are orthogonal to $O_{A} \cup \mathcal{V}_{A} \cup O_{B} \cup \mathcal{V}_{B}, O_{A} \cup \mathcal{V}_{A} \cup \mathcal{V}_{B}$, and $O_{A} \cup \mathcal{V}_{A}$, respectively. This is illustrated in Fig. 1 .

The "job" of the F12 correction, $E_{\mathrm{F} 12}$, is to correct for the BSIE of the conventional RIMP2 calculation. Since the BSIE is larger for UC monomer energies than for SNOOP monomer energies, $E_{\mathrm{F} 12}$ for each monomer will generally be more negative for UC than for SNOOP. Similarly, $E_{\mathrm{F} 12}$ will generally be more negative for SNOOP than for CP. After
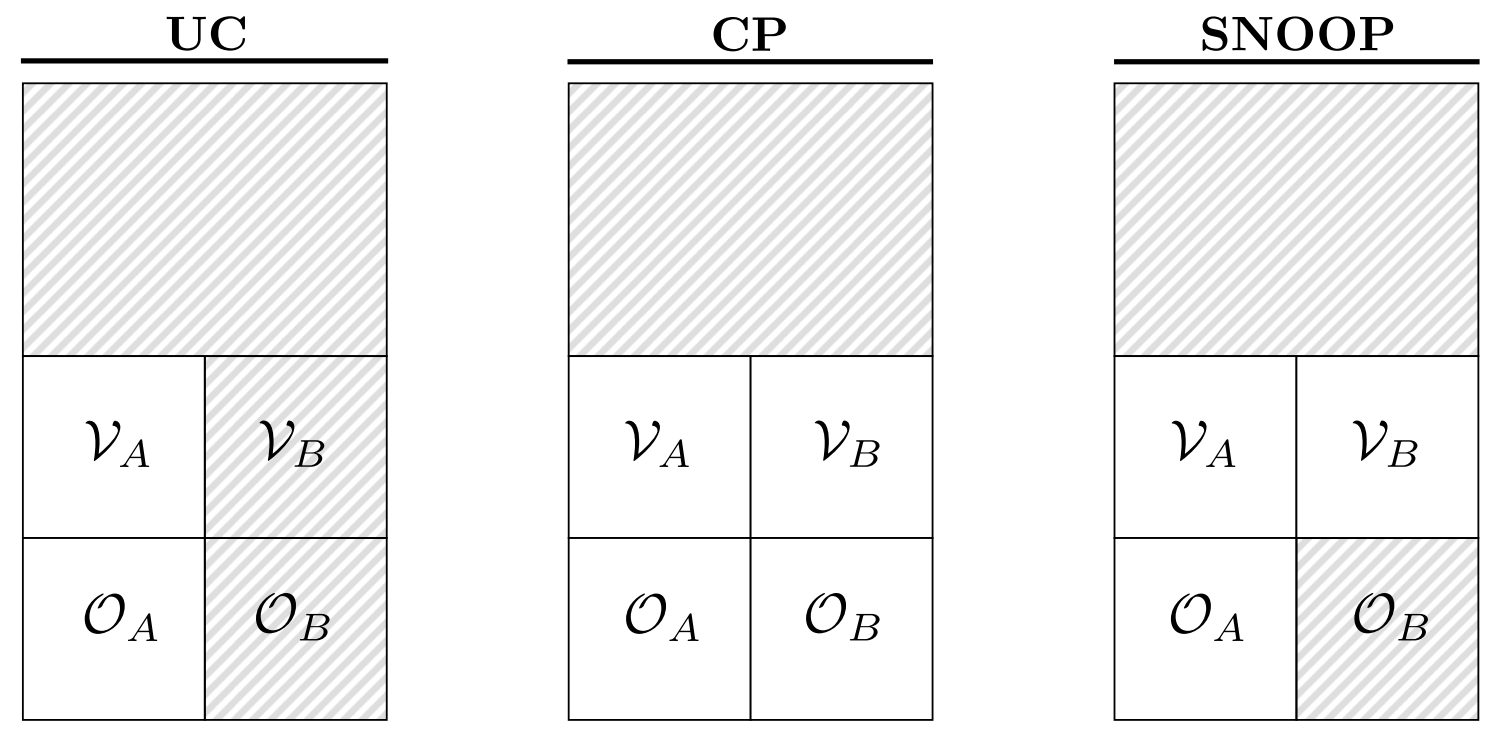

FIG. 1. A comparison of the orbital spaces employed for monomer $A$ of the UC, CP, and SNOOP approaches. White boxes denote the MO space where the conventional wave function calculation is carried out, while hatched boxes represent the complementary virtual space, $\tilde{V}_{A}$, which the F12 correction approximately describes. 
the addition of the F12 correction to the RIMP2 energy in Eq. (7), the relative ordering of interaction energies predicted by Eq. (6) may no longer hold. Nonetheless, for the practical calculations in Section IV, the ordering in Eq. (6) is in most cases observed also for RIMP2-F12 interaction energies.

\section{COMPUTATIONAL DETAILS}

We consider RIMP2 interaction energies calculated using seven different approaches: UC, CP, and SNOOP with and without the F12 correction, as well as interaction energies obtained by extrapolation. For a given cardinal number $X(X=\mathrm{T}, \mathrm{Q}, 5)$, the correlation contribution of the extrapolated interaction energy is determined by applying the $X^{-3}$ extrapolation scheme ${ }^{65-67}$ to the correlation contribution of the aug-cc-pV(X-1)Z and aug-cc-pVXZ interaction energies. Since we use a two-point extrapolation scheme, the extrapolation of the interaction energy is equivalent to performing extrapolations of the individual monomer and dimer energies. ${ }^{67}$ For the HF contribution we simply use the HF interaction energy calculated using the augcc-pVXZ basis set, since HF extrapolation schemes are often unstable. ${ }^{68}$ For the extrapolated values, we consider only CPcorrected interaction energies since their basis set convergence is in general more stable than is the case for interaction energies calculated using $\mathrm{UC}^{67}$ or SNOOP. ${ }^{29}$ Extrapolated values determined in this manner will be abbreviated EXTR in the following.

The different combinations of basis sets used in this work are listed in Table I. For the analysis of the numerical results we use the notation M-F12/XZ where M is the method (UC, $\mathrm{CP}$, SNOOP, or EXTR), the "-F12" part is used only when the F12 correction is applied, and $\mathrm{X}$ denotes the cardinal number of the regular aug-cc-pVXZ basis set with the associated RI basis set and complementary auxiliary basis set (CABS) basis set given in Table I. For example, CP-F12/TZ and CP/TZ refer to $\mathrm{CP}$-corrected interaction energies calculated using the aug-cc-pVTZ regular basis set with and without the F12 correction, respectively.

All calculations used the frozen core approximation and were carried out using a local version of the LSDALTON program. ${ }^{74,75}$ Specifically, we note that while the F12 implemen- tation currently exists only in a local version of the program, the RIMP2 method as well as the SNOOP and CP schemes are available in the latest LSDALTon release. ${ }^{75}$ The F12 correlation correction was implemented using approximation $3 C^{58,59}$ and the fixed amplitude ansatz, ${ }^{60}$ and the RI approximation was used for all F12 integrals. ${ }^{55}$ In addition, all reported F12 results use the F12 singles correction, which effectively corrects for the basis incompleteness of the Hartree-Fock energy. ${ }^{76}$ For the timings presented in Section IV B, each calculation used five nodes (each with 2 hexcore Intel/Westmere X5650 2.6 GHz, 48 GB memory and 1 TB disk) connected with QDR Infiniband (40 Gbit/s). The calculations were performed at the Centre for Scientific Computing, Aarhus, ${ }^{77}$ using Message Passage Interface (MPI) parallelization across the nodes and using Open Multi-Processing (OpenMP) parallelization across the cores on each node.

The molecular dimer geometries used were obtained from the S22 test set presented by Jurečka et al. ${ }^{47}$ which is designed to represent non-covalent interactions in biological molecules in a balanced way. As reference values for interaction energies for the 22 systems, we have used the improved numbers of Podeszwa et al. ${ }^{78}$ which employ augmented functions for all systems and larger basis sets than in the original work. For the study of clusters consisting of more than two molecules, we consider a cluster of 16 water molecules using the geometry of Yoo et al. ${ }^{79}$ (the 4444-a structure using their nomenclature) as well as a cluster of 9 methane molecules, which was built and optimized using the default settings of the Avogadro program ${ }^{80}$ (the geometry is given in the supplementary material ${ }^{84}$ ). In all the presented calculations we consider interaction energies rather than binding energies, i.e., each monomer calculation uses the geometry of the monomer in the optimized interacting system.

\section{RESULTS}

In Section IV A we present interaction energies for UC, $\mathrm{CP}$, and SNOOP with and without the F12 correction using the DZ, TZ, QZ, and $5 Z$ basis sets for four selected dimer systems from the S22 test set, two of which (water and formamide) represent dimers dominated by hydrogen bonds, and two of which (methane and ethene) represent dimers dominated by

TABLE I. Basis set combinations used for RIMP2 and RIMP2-F12 calculations: the regular AO basis sets, aug-cc-pVXZ; ${ }^{56}$ the auxiliary basis sets used for the RI approximation, aug-cc-pVXZ-RI ${ }^{69}$ and aug-cc-pwCVXZ$\mathrm{RI}$, which is build as the union of the three basis sets cc-pVXZ-RI, ${ }^{64,70}$ aug-cc-pVXZ-RI diffuse, ${ }^{69}$ and cc-pwCVXZ-RI tight; ${ }^{71}$ the complementary auxiliary basis sets (CABS) used for the F12 correction, aug-ccpVXZ_OPTRI. ${ }^{64,70,72,73}$ The $\gamma$ factor in Eq. (11) was chosen according to the recommendations of Ref. 64 .

\begin{tabular}{lllll}
\hline \hline Model & \multicolumn{1}{c}{ Regular } & RI & CABS & $\gamma$ \\
\hline RIMP2 & aug-cc-pVDZ & aug-cc-pVDZ-RI & $\ldots$ & $\ldots$ \\
& aug-cc-pVTZ & aug-cc-pVTZ-RI & $\ldots$ & $\ldots$ \\
& aug-cc-pVQZ & aug-cc-pVQZ-RI & $\ldots$ & $\ldots$ \\
& aug-cc-pV5Z & aug-cc-pV5Z-RI & $\ldots$ & $\ldots$ \\
RIMP2-F12 & aug-cc-pVDZ & aug-cc-pwCVTZ-RI & aug-cc-pVDZ_OPTRI & 1.1 \\
& aug-cc-pVTZ & aug-cc-pwCVQZ-RI & aug-cc-pVTZ_OPTRI & 1.2 \\
& aug-cc-pVQZ & aug-cc-pwCV5Z-RI & aug-cc-pVQZ_OPTRI & 1.4 \\
& aug-cc-pV5Z & aug-cc-pV6Z-RI & aug-cc-pV5Z_OPTRI & 1.4 \\
\hline \hline
\end{tabular}


TABLE II. Interaction energies in $\mathrm{kcal} / \mathrm{mol}$ for the water, formamide, methane, and ethene dimers calculated using the UC, CP, and SNOOP schemes with/without the F12 correction as well as extrapolated results.

\begin{tabular}{lccccccc}
\hline \hline Water & UC & UC-F12 & CP & CP-F12 & SNOOP & SNOOP-F12 & EXTR \\
\hline DZ & -5.210 & -5.057 & -4.366 & -4.872 & -4.633 & -4.912 & $\ldots$ \\
TZ & -5.164 & -5.016 & -4.688 & -4.943 & -4.825 & -4.960 & -4.832 \\
QZ & -5.092 & -4.991 & -4.856 & -4.957 & -4.898 & -4.960 & -4.952 \\
$5 Z$ & -5.036 & -4.972 & -4.904 & -4.960 & -4.922 & -4.962 & -4.955 \\
\hline Formamide & UC & UC-F12 & CP & CP-F12 & SNOOP & SNOOP-F12 & EXTR \\
\hline DZ & -16.157 & -15.934 & -13.953 & -15.581 & -14.722 & -15.677 & $\ldots$ \\
TZ & -16.281 & -15.924 & -15.030 & -15.765 & -15.321 & -15.800 & -15.457 \\
QZ & -16.083 & -15.856 & -15.495 & -15.796 & -15.578 & -15.802 & -15.772 \\
$5 Z$ & -15.961 & -15.826 & -15.639 & -15.800 & -15.673 & -15.803 & -15.788 \\
\hline Methane & UC & UC-F12 & CP & CP-F12 & SNOOP & SNOOP-F12 & EXTR \\
\hline DZ & -0.919 & -0.645 & -0.391 & -0.475 & -0.502 & -0.493 & $\ldots$ \\
TZ & -0.597 & -0.514 & -0.459 & -0.487 & -0.495 & -0.491 & -0.491 \\
QZ & -0.534 & -0.495 & -0.478 & -0.490 & -0.489 & -0.491 & -0.492 \\
$5 Z$ & -0.507 & -0.493 & -0.485 & -0.492 & -0.487 & -0.491 & -0.492 \\
\hline Ethene & UC & UC-F12 & CP & CP-F12 & SNOOP & SNOOP-F12 & EXTR \\
\hline DZ & -2.101 & -1.753 & -1.177 & -1.516 & -1.469 & -1.574 & $\ldots$ \\
TZ & -1.876 & -1.637 & -1.463 & -1.570 & -1.554 & -1.584 & -1.584 \\
QZ & -1.705 & -1.597 & -1.533 & -1.577 & -1.554 & -1.579 & -1.583 \\
$5 Z$ & -1.626 & -1.585 & -1.555 & -1.579 & -1.561 & -1.579 & -1.578 \\
\hline \hline
\end{tabular}

dispersion forces. This investigation allows us to estimate the errors of the EXTR/QZ and EXTR/5Z reference values of the S22 test set, ${ }^{78}$ which will prove to be useful in Section IV B where we analyze $\mathrm{UC} / \mathrm{XZ}, \mathrm{CP} / \mathrm{XZ}$, and $\mathrm{SNOOP} / \mathrm{XZ}(\mathrm{X}=\mathrm{D}$, $\mathrm{T}, \mathrm{Q})$ as well as UC-F12/DZ, CP-F12/DZ, and SNOOPF12/DZ results for the entire S22 test set. In Section IV C we consider $\mathrm{UC} / \mathrm{XZ}, \mathrm{CP} / \mathrm{XZ}$, and $\mathrm{SNOOP} / \mathrm{XZ}(\mathrm{X}=\mathrm{D}, \mathrm{T}, \mathrm{Q})$ as well as UC-F12/YZ, CP-F12/YZ, and SNOOP-F12/YZ $(\mathrm{Y}=\mathrm{D}, \mathrm{T})$ results for molecular clusters containing more than two interacting molecules, using $\left(\mathrm{H}_{2} \mathrm{O}\right)_{16}$ and $\left(\mathrm{CH}_{4}\right)_{9}$ as representative examples of clusters dominated by hydrogen bonds and dispersion effects, respectively.

We note that the RI error has been shown to be negligible compared to other error sources. ${ }^{69,78,81}$ This is also substantiated by Tables I and II in the supplementary material, ${ }^{84}$ which contain RI errors (RIMP2 vs. MP2) and

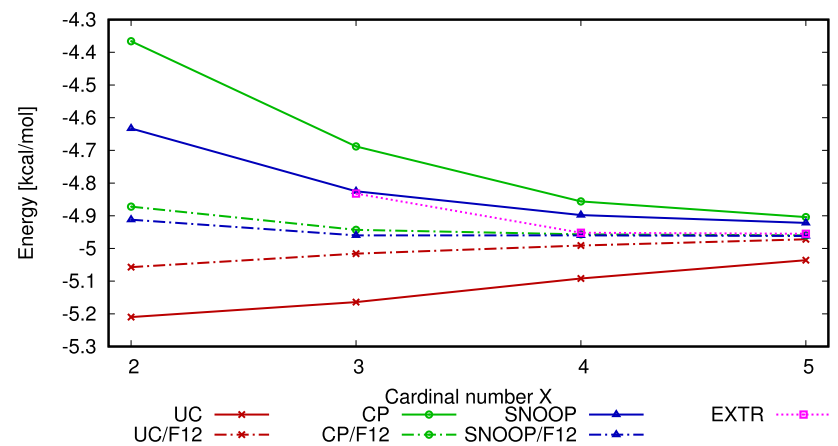

FIG. 2. Interaction energies of the water dimer with changing cardinal number. Calculated with UC, CP, and SNOOP schemes with/without the F12 correction as well as extrapolated results.
MP2 errors, respectively, of UC/XZ, CP/XZ, and SNOOP/XZ $(\mathrm{X}=\mathrm{D}, \mathrm{T}, \mathrm{Q}, 5)$ calculations (compared to EXTR/5Z results). In all cases, the RI error is more than an order of magnitude smaller than the MP2 error with the given basis, and the use of the RI approximation throughout the following analysis is thus well-justified.

\section{A. Approaching the complete basis set limit}

The RIMP2 and RIMP2-F12 results for the UC, CP, and SNOOP schemes using DZ, TZ, QZ, and $5 Z$ basis sets are given in Table II and plotted in Figs. 2-5. As expected, the results for all calculation schemes converge towards the true (and unknown) CBS value. In the following analysis, we assume that the EXTR/5Z values provide a good estimate of the CBS value, and we compare the obtained results to

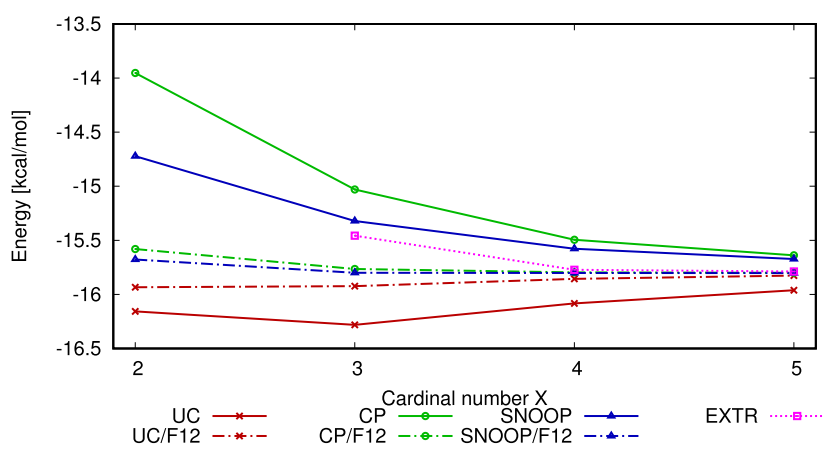

FIG. 3. Interaction energies of the formamide dimer with changing cardinal number. Calculated with UC, CP, and SNOOP schemes with/without the F12 correction as well as extrapolated results. 


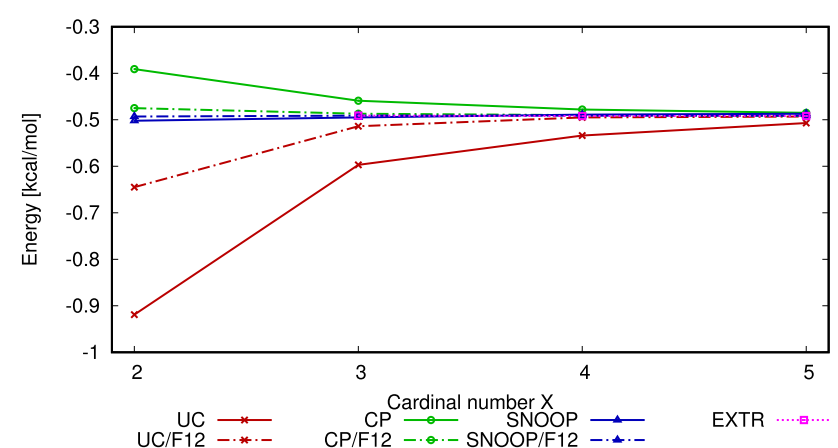

FIG. 4. Interaction energies of the methane dimer with changing cardinal number. Calculated with UC, CP, and SNOOP schemes with/without the F12 correction as well as extrapolated results.

these. For each model $\mathrm{M}$ and basis XZ we have calculated the relative deviations in percentages from the EXTR/5Z value,

$$
\delta_{\mathrm{M} / \mathrm{XZ}}=\frac{\Delta E_{A B}^{\mathrm{M} / \mathrm{XZ}}-\Delta E_{A B}^{\mathrm{EXTR} / 5 \mathrm{Z}}}{\left|\Delta E_{A B}^{\mathrm{EXTR} / 5 \mathrm{Z}}\right|} \cdot 100 \% .
$$

With this convention, a negative/positive deviation indicates that the calculated interaction energy is more/less negative than the EXTR/5Z value. The results are given in Table III. We emphasize, however, that in general we do not know whether applying the $\mathrm{X}^{-3}$ extrapolation or the F12 correction yields the more accurate estimate of the CBS value. For example, CP-F12/5Z or SNOOP-F12/5Z results may be just as accurate as or more accurate than the EXTR/5Z values. The larger deviations in Table III may thus be interpreted as

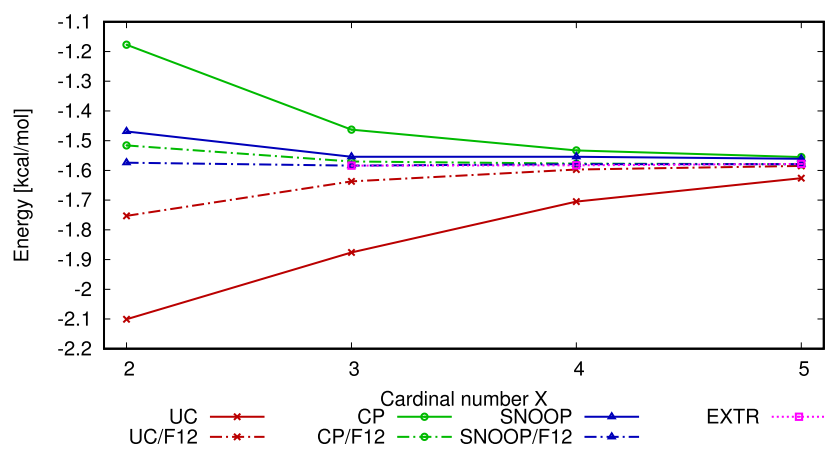

FIG. 5. Interaction energies of the ethene dimer with changing cardinal number. Calculated with UC, CP, and SNOOP schemes with/without the F12 correction as well as extrapolated results.

a good approximation of the actual errors compared to the CBS values, while the smaller deviations reflect the accuracy of the EXTR/5Z reference data. For example, the EXTR $/ 5 Z$, CP-F12/5Z, and SNOOP-F12/5Z results differ by at most $0.2 \%$, which may be viewed as a measure of the errors of these numbers compared to the true CBS value. For this reason we use the term deviation from the EXTR/5Z value rather than error.

With the exception of DZ results for the water and formamide dimers, the SNOOP results in Table III are more accurate than the UC and CP results, and they also appear more uniform for the four dimers. Comparing the UC-F12, CP-F12, and SNOOP-F12 results, the SNOOP-F12 deviations are again seen to be more uniformly small, while the UCF12 results show slower convergence and greater deviations than the CP-F12 and SNOOP-F12 numbers, particularly for

TABLE III. Relative deviations (Eq. (15)) given in percentages of the interaction energies from the EXTR/5Z values for the water, formamide, methane, and ethene dimers calculated using the UC, $\mathrm{CP}$, and SNOOP schemes with/without the F12 correction as well as EXTR/TZ and EXTR/QZ values.

\begin{tabular}{lccccccc}
\hline \hline Water & UC & UC-F12 & CP & CP-F12 & SNOOP & SNOOP-F12 & EXTR \\
\hline DZ & -5.1 & -2.1 & 11.9 & 1.7 & 6.5 & 0.9 & $\ldots$ \\
TZ & -4.2 & -1.2 & 5.4 & 0.2 & 2.6 & -0.1 & 2.5 \\
QZ & -2.8 & -0.7 & 2.0 & -0.03 & 1.2 & -0.1 & 0.1 \\
$5 Z$ & -1.6 & -0.4 & 1.0 & -0.1 & 0.7 & -0.1 & (Ref) \\
\hline Formamide & UC & UC-F12 & CP & CP-F12 & SNOOP & SNOOP-F12 & EXTR \\
\hline DZ & -2.3 & -0.9 & 11.6 & 1.3 & 6.8 & 0.7 & $\ldots$ \\
TZ & -3.1 & -0.9 & 4.8 & 0.1 & 3.0 & -0.1 & 2.1 \\
QZ & -1.9 & -0.4 & 1.9 & -0.1 & 1.3 & -0.1 & 0.1 \\
$5 Z$ & -1.1 & -0.2 & 0.9 & -0.1 & 0.7 & -0.1 & (Ref) \\
\hline Methane & UC & UC-F12 & CP & CP-F12 & SNOOP & SNOOP-F12 & EXTR \\
\hline DZ & -86.6 & -31.1 & 20.6 & 3.6 & -2.0 & -0.2 & $\ldots$ \\
TZ & -21.2 & -4.3 & 6.8 & 1.0 & -0.5 & 0.2 & 0.3 \\
QZ & -8.4 & -0.6 & 2.9 & 0.5 & 0.6 & 0.3 & 0.1 \\
$5 Z$ & -2.9 & -0.1 & 1.4 & 0.1 & 1.0 & 0.2 & (Ref) \\
\hline Ethene & UC & UC-F12 & CP & CP-F12 & SNOOP & SNOOP-F12 & EXTR \\
\hline DZ & -33.1 & -11.0 & 25.4 & 4.0 & 6.9 & 0.2 & $\ldots$ \\
TZ & -18.9 & -3.7 & 7.3 & 0.5 & 1.6 & -0.3 & -0.3 \\
QZ & -8.0 & -1.2 & 2.9 & 0.1 & 1.6 & -0.1 & -0.3 \\
$5 Z$ & -3.0 & -0.4 & 1.5 & -0.04 & 1.1 & -0.05 & (Ref) \\
\hline \hline
\end{tabular}


the dimers dominated by dispersion. However, we note that the SNOOP-F12 and CP-F12 deviations for TZ and higher basis sets are very small, and they are probably as accurate as (or more accurate than) the reference EXTR/5Z values. An error analysis of the F12 results based on Table III is therefore useful only for the $\mathrm{DZ}$ basis, where we observe that SNOOP-F12 outperforms UC-F12 and CP-F12 for all four systems. In general, the F12 correction improves the results immensely, since the CP-F12/DZ and SNOOP-F12/DZ results are as good as-if not better than - the corresponding CP/QZ and $\mathrm{SNOOP} / \mathrm{QZ}$ results.

Considering an UC calculation as the starting point, the description of the interaction energy may be improved by attempting to correct for BSSE (using either SNOOP or CP) and/or by attempting to correct for the remaining BSIE by applying the F12 correction. For the water and formamide dimers and a given choice of basis set, applying only the F12 correction (UC-F12) yields a better improvement than applying the CP or SNOOP scheme (Table III). For the methane and ethene dimers, this is also seen for QZ and $5 \mathrm{Z}$ basis sets; however, for these two systems, going from UC/XZ to $\mathrm{SNOOP} / \mathrm{XZ}(\mathrm{X}=\mathrm{D}, \mathrm{T})$ yields a more accurate result than applying the F12 correction alone (UC-F12/XZ). For methane, the $\mathrm{CP} / \mathrm{DZ}$ value is also more accurate than UC-F12/DZ. In short, it appears that for dispersion-dominated systems and small basis sets, correcting for BSSE improves the results more than applying the F12 correction, while for hydrogen-bonded systems and/or larger basis sets, the F12 correction improves the UC result more than correcting for BSSE.

In Section IV B, we will be comparing results for the entire S22 test set with the EXTR/QZ and EXTR/5Z reference values presented by Podeszwa et $a l^{78}$ To form an estimate of the accuracy of the EXTR/QZ and EXTR/5Z values as approximations to the CBS limit, we consider the difference between EXTR and SNOOP-F12 values at the QZ and 5Z basis set levels. Using our results for the four systems studied in this section, we determine

$$
\delta_{\mathrm{est}}^{\mathrm{XZ}}=\left|\frac{\Delta E_{A B}^{\mathrm{SNOOP}-\mathrm{F} 12 / \mathrm{XZ}}-\Delta E_{A B}^{\mathrm{EXTR} / \mathrm{XZ}}}{\Delta E_{A B}^{\mathrm{EXTR} / \mathrm{XZ}}}\right| \cdot 100 \%
$$

for each of the systems. The use of the error measure in Eq. (16) is justified by the logic that the SNOOP-F12 and EXTR constitute two different ways of approximating the CBS limit value, both in terms of the description of BSSE and BSIE. Specifically, recalling that the EXTR values are determined based on $\mathrm{CP}$ results, we note that the $\mathrm{CP}$ and SNOOP schemes seek to correct for the BSSE in two different manners (see Fig. 1), while the $\mathrm{X}^{-3}$ extrapolation and the F12 correction may be viewed as two different attempts at correcting for the BSIE present with the given basis set. The calculated error estimates calculated using Eq. (16) are given for the four systems in Table IV and will be used in Section IV B.

We note here that some of our EXTR/5Z results deviate from the results presented by Podeszwa et $a l^{78}$ by at most $0.001 \mathrm{kcal} / \mathrm{mol}$ (also when we do not employ the RI approximation), noting that their values are presented
TABLE IV. Estimated relative errors of EXTR/QZ and EXTR/5Z values calculated using Eq. (16).

\begin{tabular}{lcc}
\hline \hline System & QZ & $5 Z$ \\
\hline Water dimer & $0.2 \%$ & $0.1 \%$ \\
Formamide dimer & $0.2 \%$ & $0.1 \%$ \\
Methane dimer & $0.2 \%$ & $0.2 \%$ \\
Ethene dimer & $0.3 \%$ & $0.05 \%$ \\
\hline \hline
\end{tabular}

in $\mathrm{kcal} / \mathrm{mol}$ and with three decimal places. This minor discrepancy may probably be attributable to a difference in the thresholds used in the calculations, and we will disregard it in the following discussion, since it is negligible compared to the other errors considered. For consistency we will use the reference values of Podeszwa et al. ${ }^{78}$ in Section IV B.

\section{B. S22 test set}

We have calculated interaction energies with the UC/XZ, $\mathrm{CP} / \mathrm{XZ}$, and $\mathrm{SNOOP} / \mathrm{XZ}$ schemes $(\mathrm{XZ}=\mathrm{DZ}, \mathrm{TZ}, \mathrm{QZ})$ as well as with UC-F12/DZ, CP-F12/DZ, and SNOOP-F12/DZ for the $\mathrm{S} 22$ test set. ${ }^{47}$ As discussed in Section IV A, we compare the results to the improved reference values presented by Podeszwa et al. ${ }^{78}$ which are based on extrapolation of CP MP2 results as an approximation of the CBS limit values. For a model $\mathrm{M}$ and basis $\mathrm{XZ}$, the errors, $\delta_{\mathrm{M} / \mathrm{XZ}}$, compared to the interaction energies presented by Podeszwa et al., $\Delta E_{A B}^{\mathrm{REF}}$, are determined as

$$
\delta_{\mathrm{M} / \mathrm{XZ}}=\frac{\Delta E_{A B}^{\mathrm{M} / \mathrm{XZ}}-\Delta E_{A B}^{\mathrm{REF}}}{\left|\Delta E_{A B}^{\mathrm{REF}}\right|} \cdot 100 \% .
$$

The errors calculated using Eq. (17) are listed in Table V. In the work of Podeszwa et al. some interaction energies are determined at the EXTR/5Z level, while others are only determined at the EXTR/QZ level. This is reflected in Table $\mathrm{V}$ in that the values given in the $\max \left(\delta_{\text {est }}^{\mathrm{XZ}}\right)$ column are the maximum estimated errors presented in Section IV A corresponding to the basis set level of the reference calculation ( $0.3 \%$ for QZ reference data and $0.2 \%$ for $5 \mathrm{Z}$ reference data). The $\max \left(\delta_{\text {est }}^{\mathrm{XZ}}\right)$ values thus provide rather conservative estimates of the errors of the reference data. Hence, deviations from the reference values that are significantly larger than $\max \left(\delta_{\text {est }}^{\mathrm{XZ}}\right)$ may be interpreted as actual errors compared to the CBS limit, while deviations that are similar to or smaller than $\max \left(\delta_{\mathrm{est}}^{\mathrm{XZ}}\right)$ may be equally good approximations to the true CBS result and reflect the uncertainty of the EXTR/XZ reference value.

The statistical measures of maximum absolute error, mean absolute error, mean error, and standard deviations for each calculation method used are also listed in Table V. The usual trend of the UC scheme giving values that are too negative and the $\mathrm{CP}$ scheme giving values that are too positive can be seen from the results. The SNOOP and SNOOP-F12 methods produce interaction energies with positive as well as negative deviations from the CBS limit value.

Comparing the measures of maximum absolute error, $\max \left(\left|\delta_{\mathrm{M}(-\mathrm{F} 12) / \mathrm{XZ}}\right|\right)$, and mean absolute error, $\overline{\left|\delta_{\mathrm{M}(-\mathrm{F} 12) / \mathrm{XZ}}\right| \text {, for }}$ the UC, CP, and SNOOP calculations, it is consistently the case that both measures are lowest for SNOOP calculations 
TABLE V. S22 benchmark set. ${ }^{47}$ Relative deviations are given in percentages compared to results by Podeszwa et al. ${ }^{78}$ and the estimated error of the reference value, $\max \left(\delta_{\text {est }}^{\mathrm{XZ}}\right)$, is taken from Table IV according to the $\mathrm{XZ}$ basis set used for calculating the reference value.

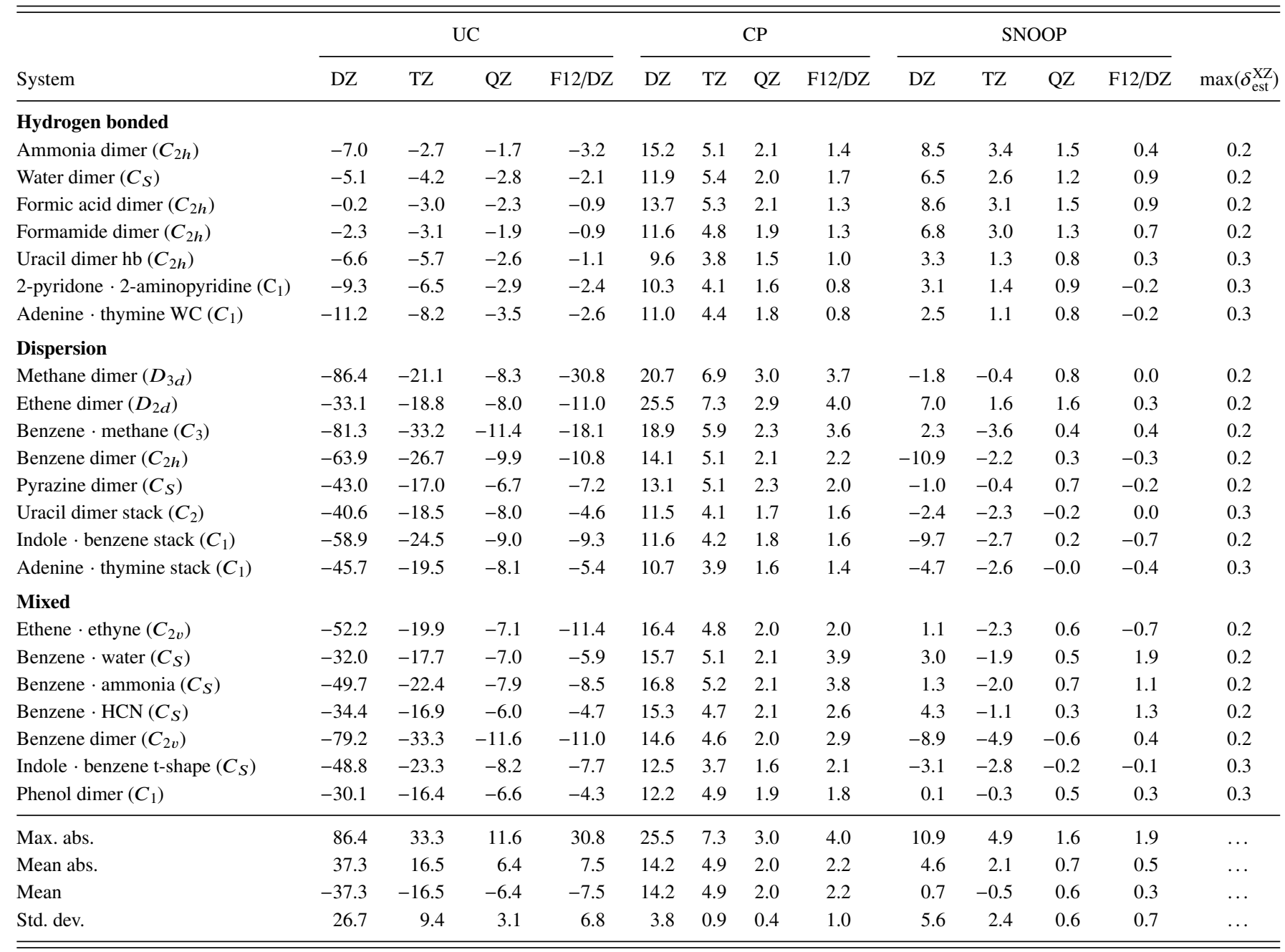

and lower for $\mathrm{CP}$ calculations than for UC calculations, i.e.,

$$
\begin{aligned}
\max \left(\left|\delta_{\mathrm{SNOOP}(-\mathrm{F} 12) / \mathrm{XZ}}\right|\right) & <\max \left(\left|\delta_{\mathrm{CP}(-\mathrm{F} 12) / \mathrm{XZ}}\right|\right) \\
& <\max \left(\mid \delta_{\mathrm{UC}(-\mathrm{F} 12) / \mathrm{XZ} \mid)}\right.
\end{aligned}
$$

and

$\overline{\left|\delta_{\mathrm{SNOOP}(-\mathrm{F} 12) / \mathrm{XZ}}\right|}<\overline{\left|\delta_{\mathrm{CP}(-\mathrm{F} 12) / \mathrm{XZ}}\right|}<\overline{\mid \delta_{\mathrm{UC}(-\mathrm{F} 12) / \mathrm{XZ}}}$.

Thus, for a given basis, the SNOOP method in general yields smaller errors than the UC and CP approaches. In particular, for the F12 results, we note that the SNOOP absolute error is smaller than the corresponding $\mathrm{UC}$ or $\mathrm{CP}$ errors for all 22 systems, except for the formic acid dimer where UC-F12 and SNOOP-F12 both display an (absolute) error of $0.9 \%$. Furthermore, it is quite remarkable that the SNOOP errors are smaller than the corresponding $\mathrm{CP}$ errors for all of the 88 reported values in Table $\mathrm{V}$ (DZ, TZ, QZ, and F12/DZ results for 22 molecules). The SNOOP results also display significantly smaller average errors than the corresponding UC and $\mathrm{CP}$ results, indicating that the SNOOP errors are rather uniformly distributed around zero. However, the standard deviation of the $\mathrm{CP} / \mathrm{XZ}$ results is smaller than those of the $\mathrm{UC} / \mathrm{XZ}$ and $\mathrm{SNOOP} / \mathrm{XZ}$ results.
The systematically positive errors of the $\mathrm{CP}$ approach are consistent with the fact that the (negative) dispersion component of the interaction energy tend to be underestimated in finite basis set calculations, as discussed in Section II A. The SNOOP scheme yields a mixture of positive and negative errors for the individual molecules and larger standard deviations than the $\mathrm{CP}$ scheme. The reason for this irregularity of the SNOOP results is explained in detail in Ref. 29, and it may be rationalized by considering the calculation of monomer $A$, which is depicted schematically in Fig. 1. When the basis set of monomer $A$ approaches completeness, $O_{A} \cup \mathcal{V}_{A}$ will also span the $O_{B}$ space, and the SNOOP and CP schemes become equivalent. This is not the case for finite basis sets, and, as the cardinal number $X$ of the aug-cc-pVXZ basis sets ${ }^{56}$ is increased, the $O_{A} \cup \mathcal{V}_{A}$ space will span an increasing part of the $O_{B}$ space. Unlike the basis set for the dimer calculation, the basis set for each of the SNOOP monomer calculations is therefore not improved systematically when the cardinal number is increased, and the SNOOP interaction energy does in general not exhibit a stable convergence with increasing cardinal number. This is to be contrasted with the $\mathrm{CP}$ scheme where the $O_{B}$ space is included in the calculation for monomer $A$ (and vice versa). The improvement of the monomer and dimer energies 
TABLE VI. Timings (in seconds) for SNOOP calculations of water dimer, benzene $\cdot$ water dimer, and adenine thymine WC dimer. As a measure of system size we also give the number of atoms in the dimer system with the number of non-hydrogen atoms given in parenthesis.

\begin{tabular}{lrrrrr}
\hline \hline System & \multicolumn{1}{c}{ DZ } & \multicolumn{1}{c}{ TZ } & \multicolumn{1}{c}{ QZ } & F12/DZ & Number of atoms \\
\hline Water dimer $\left(C_{S}\right)$ & 5 & 34 & 278 & 50 & $6(2)$ \\
Benzene $\cdot$ water $\left(C_{S}\right)$ & 104 & 1218 & 13323 & 1376 & $15(7)$ \\
Adenine $\cdot$ thymine WC $\left(C_{1}\right)$ & 1522 & 18006 & 212655 & 27493 & $30(19)$ \\
\hline \hline
\end{tabular}

upon increasing the basis set is therefore more uniform, which results in a relatively systematic convergence of the $\mathrm{CP}$ interaction energies. Consequently, interaction energies calculated using the $\mathrm{CP}$ scheme are well-suited for use in extrapolation schemes, while SNOOP interaction energies are not. Nonetheless, if the goal is to carry out a single-point calculation with a given basis set which reproduces the CBS limit value as closely as possible, then Table V shows that the SNOOP scheme generally yields smaller errors than the CP scheme.

Considering the importance of the F12 correction for each scheme, it is seen that the QZ and F12/DZ results are of similar quality for the CP and SNOOP approaches, while the UC/QZ results are somewhat better than the UCF12/DZ results (particularly for the maximum errors). It is interesting to compare the computational efforts of SNOOP calculations with and without the F12 correction. For this purpose we report timings for SNOOP calculations of three selected systems of different sizes (water dimer, benzene - water dimer, and adenine - thymine WC) in Table VI using the architecture specifications listed in Section III. The timings include the dimer calculation and the two monomer calculations required to determine the SNOOP interaction energy. Table VI shows that the computational requirements of the SNOOP/TZ and SNOOP-F12/DZ calculations are similar, while the SNOOP/QZ calculations are roughly an order of magnitude more expensive than the SNOOPF12/DZ calculations. Clearly, the best compromise between computational efforts and accuracy is obtained with the SNOOP-F12/DZ method. In fact, since the computational cost is similar for SNOOP and CP calculations, it follows from Tables V and VI that the SNOOP-F12/DZ method yields results that are significantly better than what is obtained using the CP/QZ method at roughly one tenth of the computational cost. We also note that, according to Table III, SNOOPF12/DZ results are more accurate than what is obtained by performing a DZ-TZ extrapolation of $\mathrm{CP}$ results (the cost of which is similar to SNOOP-F12/DZ).

Finally, we emphasize again that the $\max \left(\delta_{\text {est }}^{\mathrm{XZ}}\right)$ column in Table $\mathrm{V}$ is an estimate of the uncertainty of the reference value, and a calculation with a deviation that lies within the deviation interval $\pm \max \left(\delta_{\text {est }}^{\mathrm{XZ}}\right)$ may be just as accurate as the reference value. In eight cases does the SNOOP-F12/DZ calculation lie within the $\pm \max \left(\delta_{\text {est }}^{\mathrm{XZ}}\right)$ interval, while this happens four times for the SNOOP/QZ results and rarely for the other methods (and never for any of the $\mathrm{CP}$ results). This also highlights the accuracy of the SNOOP results.

\section{Interaction energies of molecular clusters}

Here we present results for interaction energies of two molecular clusters, $\left(\mathrm{H}_{2} \mathrm{O}\right)_{16}$ and $\left(\mathrm{CH}_{4}\right)_{9}$, for the UC/XZ, $\mathrm{CP} / \mathrm{XZ}, \mathrm{SNOOP} / \mathrm{XZ}$, and EXTR/XZ methods $(\mathrm{XZ}=\mathrm{DZ}$, $\mathrm{TZ}, \mathrm{QZ}$ ) as well as the UC-F12/YZ, CP-F12/YZ, and SNOOP-F12/YZ methods ( $\mathrm{YZ}=\mathrm{DZ}, \mathrm{TZ})$. The interaction energies are listed in Table VII. To enable a comparison of the results, the deviations from the EXTR/QZ values are listed in Table VIII. However, generalizing the results of Table III, we note that the SNOOP-F12/TZ results are probably as accurate or more accurate than the EXTR/QZ results. Nonetheless, the very small deviations of the SNOOP-F12/TZ and EXTR/QZ results-which represent quite different ways of approximating the true CBS value-could be interpreted as an indication that the SNOOP-F12/TZ and EXTR/QZ results are both close to the true CBS result.

From the results for the two clusters in Table VIII, it is seen that the $\mathrm{SNOOP}(-\mathrm{F} 12)$ deviations are smaller than the corresponding UC(-F12) and $\mathrm{CP}(-\mathrm{F} 12)$ deviations in all cases, except for the UC/DZ results for the $\left(\mathrm{H}_{2} \mathrm{O}\right)_{16}$ cluster. In particular, for the dispersion-dominated $\left(\mathrm{CH}_{4}\right)_{9}$ cluster, the SNOOP deviations are all within $1 \%$ relative deviation from the EXTR/QZ value.

TABLE VII. Interaction energies of the $\left(\mathrm{H}_{2} \mathrm{O}\right)_{16}$ and $\left(\mathrm{CH}_{4}\right)_{9}$ molecular clusters calculated using the $\mathrm{UC}, \mathrm{CP}$, and SNOOP schemes with/without the F12 correction as well as extrapolated values for each cluster system.

\begin{tabular}{lccccccc}
\hline \hline$\left(\mathrm{H}_{2} \mathrm{O}\right)_{16}$ & $\mathrm{UC}$ & $\mathrm{UC}-\mathrm{F} 12$ & $\mathrm{CP}$ & $\mathrm{CP}-\mathrm{F} 12$ & SNOOP & SNOOP-F12 & EXTR \\
\hline $\mathrm{DZ}$ & -177.4 & -171.7 & -146.6 & -166.5 & -153.5 & -167.0 & $\ldots$ \\
$\mathrm{TZ}$ & -176.2 & -171.2 & -160.2 & -168.9 & -162.6 & -169.2 & -165.5 \\
$\mathrm{QZ}$ & -173.3 & $\ldots$ & -165.7 & $\ldots$ & -166.2 & $\ldots$ & -169.1 \\
\hline $\left.\mathrm{CH}_{4}\right)_{9}$ & $\mathrm{UC}$ & $\mathrm{UC}-\mathrm{F} 12$ & $\mathrm{CP}$ & $\mathrm{CP}-\mathrm{F} 12$ & SNOOP & SNOOP-F12 & EXTR \\
\hline $\mathrm{DZ}$ & -9.101 & -6.314 & -3.875 & -4.685 & -4.844 & -4.868 & $\ldots$ \\
$\mathrm{TZ}$ & -6.167 & -5.125 & -4.577 & -4.835 & -4.849 & -4.868 & -4.887 \\
$\mathrm{QZ}$ & -5.417 & $\ldots$ & -4.752 & $\ldots$ & -4.829 & $\ldots$ & -4.875 \\
\hline \hline
\end{tabular}


TABLE VIII. Relative deviations in percentages from the EXTR/QZ values of calculations performed for the $\left(\mathrm{H}_{2} \mathrm{O}\right)_{16}$ and $\left(\mathrm{CH}_{4}\right)_{9}$ molecular clusters with the UC, CP, and SNOOP schemes with/without the F12 correction.

\begin{tabular}{lcccccc}
\hline \hline$\left(\mathrm{H}_{2} \mathrm{O}\right)_{16}$ & $\mathrm{UC}$ & $\mathrm{UC}-\mathrm{F} 12$ & $\mathrm{CP}$ & CP-F12 & SNOOP & SNOOP-F12 \\
\hline $\mathrm{DZ}$ & -4.9 & -1.5 & 13.3 & 1.6 & 9.2 & 1.2 \\
$\mathrm{TZ}$ & -4.2 & -1.2 & 5.3 & 0.1 & 3.8 & -0.04 \\
$\mathrm{QZ}$ & -2.5 & $\ldots$ & 2.0 & $\ldots$ & 1.7 & $\ldots$ \\
\hline$\left(\mathrm{CH}_{4}\right)_{9}$ & $\mathrm{UC}$ & $\mathrm{UC}-\mathrm{F} 12$ & $\mathrm{CP}$ & $\mathrm{CP}-\mathrm{F} 12$ & SNOOP & SNOOP-F12 \\
\hline $\mathrm{DZ}$ & -86.7 & -29.5 & 20.5 & 3.9 & 0.6 & 0.1 \\
$\mathrm{TZ}$ & -26.5 & -5.1 & 6.1 & 0.8 & 0.5 & 0.1 \\
$\mathrm{QZ}$ & -11.1 & $\cdots$ & 2.5 & $\ldots$ & 0.9 & $\cdots$ \\
\hline \hline
\end{tabular}

With the application of the F12 correction, the UC, CP, and SNOOP deviations in Table VIII all become smaller. For the $\left(\mathrm{H}_{2} \mathrm{O}\right)_{16}$ cluster, the UC-F12/DZ, CP-F12/DZ, and SNOOP-F12/DZ results are similar, while, at the TZ level, the UC-F12 deviation is an order of magnitude larger than the CP-F12 and SNOOP-F12 results, which are probably as accurate as or more accurate than the EXTR/QZ reference value. For the $\left(\mathrm{CH}_{4}\right)_{9}$ cluster, the ordering of methods by smallest deviation is the same with and without the F12 correction, i.e., SNOOP is more accurate than $\mathrm{CP}$, which, in turn, is more accurate than UC. The greater importance of correcting for BSSE in dispersion-dominated systems than in hydrogen-bonded systems is exemplified here by the fact that the UC results for the $\left(\mathrm{CH}_{4}\right)_{9}$ cluster show a much greater relative deviation than is the case for the $\left(\mathrm{H}_{2} \mathrm{O}\right)_{16}$ cluster. Furthermore, at the DZ level, applying either the CP or SNOOP scheme yields a greater improvement of the UC results for $\left(\mathrm{CH}_{4}\right)_{9}$ than applying the $\mathrm{F} 12$ correction.

It is also worth noting that the relative deviations for the $\left(\mathrm{H}_{2} \mathrm{O}\right)_{16}$ and $\left(\mathrm{CH}_{4}\right)_{9}$ clusters in Table VIII are strikingly similar to the relative deviations of the $\left(\mathrm{H}_{2} \mathrm{O}\right)_{2}$ and $\left(\mathrm{CH}_{4}\right)_{2}$ dimers in Table V for all approaches (UC, CP, and SNOOP with/without the F12 correction and for all basis sets). This indicates that the molecular dimer results of Table $\mathrm{V}$ can be generalized to more complex molecular clusters. It thus appears that SNOOP is generally preferable over UC or CP for molecular dimers and clusters and that SNOOP-F12/DZ yields the best compromise between computational cost and accuracy.

Finally, we note that the interaction energies for the $\left(\mathrm{H}_{2} \mathrm{O}\right)_{16}$ cluster presented here (e.g., $-169.2 \mathrm{kcal} / \mathrm{mol}$ for SNOOP-F12/TZ) are slightly different from the MP2 CBS estimates presented by Wang et al. ${ }^{82}(-164.1 \mathrm{kcal} / \mathrm{mol})$ and Miliordos and Xantheas ${ }^{83}(-161.9 \mathrm{kcal} / \mathrm{mol})$. The primary reason for this difference is that-in contrast to the results presented in Table VII-these works included the relaxation energy of the monomers, which leads to interaction energies that are less negative than the ones reported here.

\section{CONCLUSIONS}

We have augmented the SNOOP scheme for determining intermolecular interaction energies with an F12 correction and compared it to UC and CP results with/without the F12 correction for the molecular dimers of the S22 test set $^{47}$ as well as $\left(\mathrm{H}_{2} \mathrm{O}\right)_{16}$ and $\left(\mathrm{CH}_{4}\right)_{9}$ molecular clusters. The calculations were performed using the RIMP2 method, and the results show that, for a given aug-cc-pVXZ basis set, the SNOOP scheme in general yields smaller errors compared to the CBS limit value than the $\mathrm{UC}$ and $\mathrm{CP}$ schemes. In fact, SNOOP provides smaller errors than $\mathrm{CP}$ for all 22 molecules in the S22 test set for all the considered basis sets, regardless of whether the F12 correction is applied or not. The use of the F12 correction improves the quality of the results tremendously for all schemes, particularly SNOOP and CP. In fact, the SNOOP-F12/DZ method yields results that are comparable in quality to a SNOOP/QZ calculation or better and requires computational resources corresponding only to a SNOOP/TZ calculation. Thus, SNOOP-F12/DZ is a computationally economical way of achieving an accurate estimate of the CBS limit value of an interaction energy. For molecular clusters consisting of more than two molecules, the investigation shows the same tendencies as for the molecular dimers. Future work includes a generalization of the SNOOP-F12 method to more accurate models of the coupled cluster hierarchy as well as developments of SNOOP(-F12) methods for linear-scaling coupled-cluster approaches.

\section{ACKNOWLEDGMENTS}

The research leading to these results has received funding from the European Research Council under the European Unions Seventh Framework Programme (No. FP/20072013)/ERC Grant Agreement No. 291371. The numerical results presented in this work were performed at the Centre for Scientific Computing, Aarhus (http://phys.au.dk/forskning/ (scaa/).

\section{APPENDIX: SNOOP INTERACTION ENERGIES FOR MOLECULAR CLUSTERS}

In this section we generalize the SNOOP scheme to enable the calculation of interaction energies for molecular clusters containing more than two monomers and demonstrate that the parameter analysis summarized by Eq. (3) is still valid.

We define the interaction energy of a system consisting of $N>2$ monomers $M_{1}, M_{2}, \ldots, M_{N}$ in the same way as for $N=2$, i.e., 


$$
\Delta E_{M_{1} \cdots M_{N}}=E_{M_{1} \cdots M_{N}}-\sum_{i=1}^{N} E_{M_{i}},
$$

where $E_{M_{1} \cdots M_{N}}$ is total energy of the interacting system and $E_{M_{i}}$ is the energy of the isolated monomer $M_{i}$. The interacting system is denoted $M_{1} \cdots M_{N}$, while the noninteracting system is denoted $M_{1}+\cdots+M_{N}$.

As for dimer interaction energies, the energy of the interacting system, $E_{M_{1} \cdots M_{N}}$, is calculated in the same way for the UC, CP, and SNOOP schemes, while the calculation of monomer energies is different. For the UC approach, each monomer energy is calculated without considering the other monomers, i.e., by an energy calculation using the basis functions belonging to the particular monomer. Using the $\mathrm{CP}$ method, for each monomer $M_{i}$, the basis functions of all the other monomers are added to the monomer basis set in the energy calculation. Finally, using the SNOOP scheme, for each monomer $M_{i}$, the virtual MOs from all other UC calculations are added to the monomer basis.

For HF calculations the number of non-redundant parameters $P$ is ${ }^{29,30}$

$$
P=o \cdot v,
$$

where $o / v$ is the number of occupied/virtual MOs. The number of non-redundant HF parameters for the interacting system is thus

$$
P_{M_{1} \cdots M_{N}}=\left(\sum_{i=1}^{N} o_{i}\right) \cdot\left(\sum_{i=1}^{N} v_{i}\right)=o_{S} v_{S},
$$

where $o_{i}$ and $v_{i}$ are the number of occupied and virtual orbitals for monomer $M_{i}$, while $o_{S}$ and $v_{S}$ are the sums of the number of occupied and virtual orbitals for all monomers, respectively. The number of occupied orbitals for monomer $M_{i}$ is thus $o_{i}$, regardless of which scheme is employed, while, for the UC, CP, and SNOOP approaches, the number of virtual orbitals for monomer $M_{i}$ is $v_{i}, v_{S}$ plus the number of occupied orbitals from all other monomers, and $v_{S}$, respectively. The resulting number of non-redundant $\mathrm{HF}$ parameters for the noninteracting $M_{1}+\cdots+M_{N}$ system (the sum of the number of parameters for the $M_{1}, M_{2}, \ldots, M_{N}$ monomer calculations) thus become

$$
\begin{gathered}
P_{M_{1}+\cdots+M_{N}}^{\mathrm{UC}}=\sum_{i=1}^{N} o_{i} \cdot v_{i}, \\
P_{M_{1}+\cdots+M_{N}}^{\mathrm{SNOOP}}=\sum_{i=1}^{N} o_{i} v_{S}=o_{S} v_{S}, \\
P_{M_{1}+\cdots+M_{N}}^{\mathrm{CP}}=\sum_{i=1}^{N} o_{i} \cdot\left[v_{S}+\sum_{j=1, j \neq i}^{N} o_{j}\right] .
\end{gathered}
$$

By combining the above equations, it is seen that Eq. (3) is satisfied also for more than $N$ subsystems

$P_{M_{1}+\cdots+M_{N}}^{\mathrm{UC}}<P_{M_{1}+\cdots+M_{N}}^{\mathrm{SNOOP}}=P_{M_{1} \cdots M_{N}}<P_{M_{1}+\cdots+M_{N}}^{\mathrm{CP}}$.

Hence, the desired parameter balance for the description of the interacting system $M_{1} \cdots M_{N}$ and the noninteracting system $M_{1}+\cdots+M_{N}$ is satisfied only for the SNOOP scheme.
The above analysis was carried out for the HF optimization parameters, but a similar analysis for the correlation parameters may be performed analogously. ${ }^{29} \mathrm{We}$ note that Eq. (A7) holds for the conventional calculation, while the parameter analysis becomes somewhat ill-defined when the F12 correction is applied-in particular, when the F12 amplitudes are fixed, ${ }^{60}$ and no wave function parameters are optimized for the F12 correction. We therefore have not considered the F12 correction here, but we note that the UC, SNOOP, and CP schemes for more than two monomers may all be augmented with the F12 correction by a simple generalization of the strategy devised in Section II B.

${ }^{1}$ B. Liu and A. McLean, J. Chem. Phys. 59, 4557 (1973).

${ }^{2}$ S. F. Boys and F. Bernardi, Mol. Phys. 19, 553 (1970).

${ }^{3}$ J. R. Alvarez-Idaboy and A. Galano, Theor. Chem. Acc. 126, 75 (2010).

${ }^{4}$ L. M. Mentel and E. J. Baerends, J. Chem. Theory Comput. 10, 252 (2013).

${ }^{5} \mathrm{M}$. Gutowski, J. van Lenthe, J. Verbeek, F. van Duijneveldt, and G. Chałasiński, Chem. Phys. Lett. 124, 370 (1986).

${ }^{6}$ M. Gutowski, F. B. van Duijneveldt, G. Chałasiński, and L. Piela, Mol. Phys. 61, 233 (1987).

${ }^{7}$ G. Chałasiński and M. Gutowski, Chem. Rev. 88, 943 (1988).

${ }^{8}$ J. H. van Lenthe, J. G. C. M. van Duijneveldt-van de Rijdt, and F. B. van Duijneveldt, Adv. Chem. Phys. 69, 521 (1987).

${ }^{9}$ S. Scheiner, Reviews in Computational Chemistry (VCH, New York, 1991), p. 165.

${ }^{10}$ I. Mayer and L. Turi, J. Mol. Struct.: THEOCHEM 227, 43 (1991).

${ }^{11}$ M. Gutowski, J. G. C. M. van Duijneveldt-van de Rijdt, J. H. van Lenthe, and F. B. van Duijneveldt, J. Chem. Phys. 98, 4728 (1993).

${ }^{12}$ M. Gutowski and G. Chałasiński, J. Chem. Phys. 98, 5540 (1993).

${ }^{13}$ D. Cook, J. A. Sordo, and T. L. Sordo, Int. J. Quantum Chem. 48, 375 (1993).

${ }^{14}$ F. B. van Duijneveldt, J. G. C. M. van Duijneveldt-vande Rijdt, and J. H. van Lenthe, Chem. Rev. 94, 1873 (1994).

${ }^{15}$ R. Wieczorek, L. Haskamp, and J. J. Dannenberg, J. Phys. Chem. A 108 , 6713 (2004)

${ }^{16}$ C. S. Nash, J. Chem. Theory Comput. 1, 261 (2005).

${ }^{17}$ J. Daudey, P. Claverie, and J. Malrieu, Int. J. Quantum Chem. 8, 1 (1974).

${ }^{18}$ J. H. van Lenthe, T. van Dam, F. B. van Duijneveldt, and L. M. J. KroonBatenburg, Faraday Symp. Chem. Soc. 19, 125 (1984).

${ }^{19}$ M. Schütz, S. Brdarski, P.-O. Widmark, R. Lindh, and G. Karlström, J. Chem. Phys. 107, 4597 (1997).

${ }^{20}$ L. A. Burns, M. S. Marshall, and C. D. Sherrill, J. Chem. Theory Comput. 10, 49 (2014).

${ }^{21}$ B. Brauer, M. K. Kesharwani, and J. M. Martin, J. Chem. Theory Comput. 10, 3791 (2014).

${ }^{22}$ H. Kruse and S. Grimme, J. Chem. Phys. 136, 154101 (2012).

${ }^{23}$ B. Jeziorski and W. Kolos, Molecular Interactions (Wiley, New York, 1982), p. 1.

${ }^{24}$ B. Jeziorski, R. Moszynski, and K. Szalewicz, Chem. Rev. 94, 1887 (1994).

${ }^{25}$ I. Mayer, Int. J. Quantum Chem. 23, 341 (1983).

${ }^{26}$ J. Noga and A. Vibok, Chem. Phys. Lett. 180, 114 (1991).

${ }^{27}$ J. M. Cullen, Int. J. Quantum Chem., Symp. 25, 193 (1991).

${ }^{28}$ A. J. Sadlej, J. Chem. Phys. 95, 6705 (1991).

${ }^{29}$ K. Kristensen, P. Ettenhuber, J. J. Eriksen, F. Jensen, and P. Jørgensen, J. Chem. Phys. 142, 114116 (2015).

${ }^{30}$ T. Helgaker, P. Jørgensen, and J. Olsen, Molecular Electronic Structure Theory, 1st ed. (Wiley, Chichester, England, 2000).

${ }^{31}$ T. Kato, Commun. Pure Appl. Math. 10, 151 (1957).

${ }^{32}$ E. A. Hylleraas, Z. Phys. 54, 347 (1929).

${ }^{33}$ W. Kutzelnigg, Theor. Chim. Acta 68, 445 (1985).

${ }^{34}$ W. Kutzelnigg and W. Klopper, J. Chem. Phys. 94, 1985 (1991).

${ }^{35}$ V. Termath, W. Klopper, and W. Kutzelnigg, J. Chem. Phys. 94, 2002 (1991).

${ }^{36}$ C. Hättig, W. Klopper, A. Köhn, and D. P. Tew, Chem. Rev. 112, 4 (2012).

${ }^{37}$ L. Kong, F. A. Bischoff, and E. F. Valeev, Chem. Rev. 112, 75 (2012).

${ }^{38}$ S. Ten-no and J. Noga, Wiley Interdiscip. Rev.: Comput. Mol. Sci. 2, 114 (2012).

${ }^{39}$ O. Marchetti and H.-J. Werner, Phys. Chem. Chem. Phys. 10, 3400 (2008).

${ }^{40}$ J. R. Lane and H. G. Kjaergaard, J. Chem. Phys. 131, 034307 (2009).

${ }^{41}$ O. Marchetti and H.-J. Werner, J. Phys. Chem. A 113, 11580 (2009).

${ }^{42}$ H.-J. Werner, T. B. Adler, G. Knizia, and F. R. Manby, Recent Progress in Coupled Cluster Methods (Springer, 2010), pp. 573-619. 
${ }^{43}$ M. S. Marshall, L. A. Burns, and C. D. Sherrill, J. Chem. Phys. 135, 194102 (2011).

${ }^{44}$ J. D. McMahon and J. R. Lane, J. Chem. Phys. 135, 154309 (2011).

${ }^{45}$ K. Patkowski, J. Chem. Phys. 137, 034103 (2012).

${ }^{46}$ K. Patkowski, J. Chem. Phys. 138, 154101 (2013).

${ }^{47}$ P. Jurečka, J. Šponer, J. Černỳ, and P. Hobza, Phys. Chem. Chem. Phys. 8, 1985 (2006).

${ }^{48}$ J. L. Whitten, J. Chem. Phys. 58, 4496 (1973).

${ }^{49}$ B. I. Dunlap, J. W. D. Connolly, and J. R. Sabin, J. Chem. Phys. 71, 3396 (1979).

${ }^{50}$ B. I. Dunlap, J. W. D. Connolly, and J. R. Sabin, J. Chem. Phys. 71, 4993 (1979).

${ }^{51}$ C. Van Alsenoy, J. Comput. Chem. 9, 620 (1988).

${ }^{52}$ O. Vahtras, J. Almlöf, and M. W. Feyereisen, Chem. Phys. Lett. 213, 514 (1993).

${ }^{53}$ A. R. Kendall and A. H. Früchtl, Theor. Chem. Acc. 97, 158 (1997).

${ }^{54}$ M. Feyereisen, G. Fitzgerald, and A. Komornicki, Chem. Phys. Lett. 208, 359 (1993)

${ }^{55}$ F. R. Manby, J. Chem. Phys. 119, 4607 (2003).

${ }^{56}$ R. A. Kendall, T. H. Dunning, Jr., and R. J. Harrison, J. Chem. Phys. 96, 6796 (1992).

${ }^{57}$ W. Kutzelnigg and J. D. Morgan, J. Chem. Phys. 96, 4484 (1992).

${ }^{58}$ H.-J. Werner, T. B. Adler, and F. R. Manby, J. Chem. Phys. 126, 164102 (2007).

${ }^{59}$ S. Kedžuch, M. Milko, and J. Noga, Int. J. Quantum Chem. 105, 929 (2005).

${ }^{60}$ S. Ten-no, J. Chem. Phys. 121, 117 (2004).

${ }^{61}$ B. J. Persson and P. R. Taylor, J. Chem. Phys. 105, 5915 (1996).

${ }^{62}$ S. Ten-no, Chem. Phys. Lett. 398, 56 (2004).

${ }^{63}$ D. P. Tew and W. Klopper, J. Chem. Phys. 123, 074101 (2005).

${ }^{64}$ K. A. Peterson, T. B. Adler, and H.-J. Werner, J. Chem. Phys. 128, 084102 (2008).

${ }^{65}$ T. Helgaker, W. Klopper, H. Koch, and J. Noga, J. Chem. Phys. 106, 9639 (1997).

${ }^{66}$ A. Halkier, T. Helgaker, P. Jørgensen, W. Klopper, H. Koch, J. Olsen, and A. K. Wilson, Chem. Phys. Lett. 286, 243 (1998).

${ }^{67}$ A. Halkier, W. Klopper, T. Helgaker, P. Jørgensen, and P. R. Taylor, J. Chem. Phys. 111, 9157 (1999).

${ }^{68}$ A. Halkier, T. Helgaker, P. Jørgensen, W. Klopper, and J. Olsen, Chem. Phys. Lett. 302, 437 (1999).
${ }^{69}$ F. Weigend, A. Köhn, and C. Hättig, J. Chem. Phys. 116, 3175 (2002).

${ }^{70}$ J. G. Hill and K. A. Peterson, Phys. Chem. Chem. Phys. 12, 10460 (2010).

${ }^{71}$ C. Hättig, Phys. Chem. Chem. Phys. 7, 59 (2005).

${ }^{72}$ E. F. Valeev, Chem. Phys. Lett. 395, 190 (2004).

${ }^{73}$ W. Klopper and C. C. M. Samson, J. Chem. Phys. 116, 6397 (2002).

${ }^{74}$ K. Aidas, C. Angeli, K. L. Bak, V. Bakken, R. Bast, L. Boman, O. Christiansen, R. Cimiraglia, S. Coriani, P. Dahle, E. K. Dalskov, U. Ekström, T. Enevoldsen, J. J. Eriksen, P. Ettenhuber, B. Fernández, L. Ferrighi, H. Fliegl, L. Frediani, K. Hald, A. Halkier, C. Hättig, H. Heiberg, T. Helgaker, A. C. Hennum, H. Hettema, E. Hjertenæs, S. Høst, I.-M. Høyvik, M. F. Iozzi, B. Jansik, H. J. A. Jensen, D. Jonsson, P. Jørgensen, J. Kauczor, S. Kirpekar, T. Kjærgaard, W. Klopper, S. Knecht, R. Kobayashi, H. Koch, J. Kongsted, A. Krapp, K. Kristensen, A. Ligabue, O. B. Lutnæs, J. I. Melo, K. V. Mikkelsen, R. H. Myhre, C. Neiss, C. B. Nielsen, P. Norman, J. Olsen, J. M. H. Olsen, A. Osted, M. J. Packer, F. Pawlowski, T. B. Pedersen, P. F. Provasi, S. Reine, Z. Rinkevicius, T. A. Ruden, K. Ruud, V. Rybkin, P. Salek, C. C. M. Samson, A. S. de Merás, T. Saue, S. P. A. Sauer, B. Schimmelpfennig, K. Sneskov, A. H. Steindal, K. O. Sylvester-Hvid, P. R. Taylor, A. M. Teale, E. I. Tellgren, D. P. Tew, A. J. Thorvaldsen, L. Thøgersen, O. Vahtras, M. A. Watson, D. J. D. Wilson, M. Ziolkowski, and H. Ågren, Wiley Interdiscip. Rev.: Comput. Mol. Sci. 4, 269 (2013).

${ }^{75}$ LSDalton, a linear scaling molecular electronic structure program, Release Dalton2016 (2016), see http://daltonprogram.org.

${ }^{76}$ J. Noga and J. Šimunek, Chem. Phys. 356, 1 (2009).

${ }^{77}$ Centre for Scientific Computing, Aarhus, Denmark (http://phys.au.dk/ forskning/cscaa/).

${ }^{78}$ R. Podeszwa, K. Patkowski, and K. Szalewicz, Phys. Chem. Chem. Phys. 12, 5974 (2010).

${ }^{79}$ S. Yoo, E. Apr, X. C. Zeng, and S. S. Xantheas, J. Phys. Chem. Lett. 1, 3122 (2010).

${ }^{80}$ M. D. Hanwell, D. E. Curtis, D. C. Lonie, T. Vandermeersch, E. Zurek, and G. R. Hutchison, J. Cheminf. 4, 17 (2012).

${ }^{81}$ D. Quiñonero, C. Garau, A. Frontera, P. Ballester, A. Costa, and P. M. Deyà, J. Phys. Chem. A 109, 4632 (2005).

${ }^{82}$ F.-F. Wang, M. J. Deible, and K. D. Jordan, J. Phys. Chem. A 117, 7606 (2013).

${ }^{83}$ E. Miliordos and S. S. Xantheas, J. Chem. Phys. 142, 234303 (2015).

${ }^{84}$ See supplementary material at http://dx.doi.org/10.1063/1.4950846 for cluster geometries and RIMP2 errors. 